\title{
Excited State Absorption in the Si Nanocluster-Er Material System
}

\author{
W. H. Loh and A. J. Kenyon
}

\begin{abstract}
The issue of $\mathrm{Er}^{3+}$ excited state absorption in the $\mathrm{Si}$ nanocluster-Er material system is highlighted, and an analysis incorporating this interaction presented. We compare our analysis with a prior model of this material, and with regard to previously reported photoluminescence behavior. We show that excited state absorption can explain behavior that has previously been observed, but had not been satisfactorily accounted for.
\end{abstract}

Index Terms—Erbium, optical amplifier, silicon nanocrystal.

\section{BACKGROUND}

$\mathbf{S}$ ILICON nanocluster ( $\mathrm{nc}$ ) sensitized Er-doped silica is a material system that has generated great interest, as it holds considerable promise for practical silicon-based lasers and optical amplifiers [1], [2]. The basic appeal behind the Si nc-sensitized Er system is simple: Using $\mathrm{Si} \mathrm{nc}$ as the sensitizer to rare-earth ions enables one to take advantage of the large optical absorption cross section of silicon, which is four to five orders of magnitude larger than that of rare-earths in silica. In addition, the broad-band absorption spectrum of Si nc considerably relaxes the wavelength requirements of the pump source, allowing a wider variety of lasers, even light-emitting diodes (LEDs), to be used. Using LEDs instead of laser diodes as pump sources is extremely attractive, as it would reduce pump costs by 10- to 100-fold, extending the scope and accessibility of many more optical applications. There are numerous reports of strong 1550-nm luminescence [3]-[5] in this material system, and even some experimental reports of optical gain [1], [6] in a fabricated waveguide. However, no lasing action has been achieved to date. In spite of considerable work to understand the underlying issues and physics [7]-[9], key optimization parameters and their determining factors remain less than clear. It is the purpose of this work to point out a key interaction-excited state absorption-in this material system; by highlighting and analyzing it here, we believe it will help point the way forward to efficient silicon-based lasers and optical amplifiers.

\section{MODEL}

Fig. 1 shows a commonly accepted picture describing the $\mathrm{Si}$ nc sensitized Er system. The Si nc is excited (transition I) and its energy transferred to the Er (II), promoting an Er ion to an upper level state (III). The ion decays rapidly and nonradiatively (IV)

Manuscript received October 11, 2005; revised October 21, 2005.

W. H. Loh is with the Optoelectronics Research Centre, University of Southampton, Southampton SO17 1BJ, U.K. (e-mail: whl@orc.soton.ac.uk).

A. J. Kenyon is with the Department of Electronic and Electrical Engineering, University College London, London WC1E 7JE, U.K.

Digital Object Identifier 10.1109/LPT.2005.861266

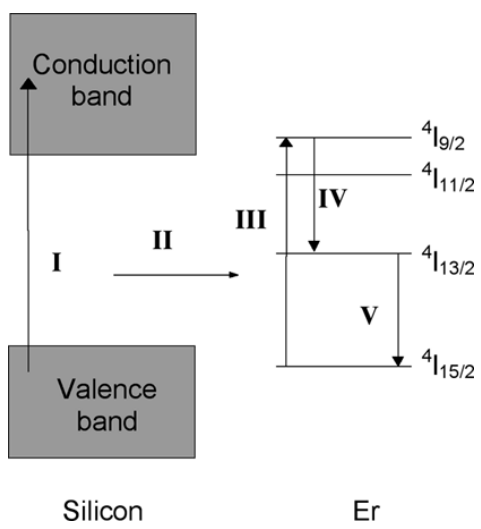

Fig. 1. Conventional picture of the Si nc-Er material system and the key interactions.

to the ${ }^{4} I_{13 / 2}$ metastable state, finally returning to the ground state via optical emission $(\mathrm{V})$.

It is generally accepted that the Er ions that are optically active and excitable by the Si nc lie in close proximity (within $1-2 \mathrm{~nm}$ ) to the nanoclusters. In many reports, the Si nanoclusters, which are themselves luminescent, are found to have their broad photoluminescence (PL) peaks located between 1.5 and $1.6 \mathrm{eV}$ (or a wavelength around $800 \mathrm{~nm}$ ), which has been attributed either to the silicon-oxygen double bond at the silicon-silica interface [10], [11] or to radiative recombination of confined excitons [12]. Regardless of the cause, spectral hole-burning studies strongly indicate that resonant energy transfer does occur from the $\mathrm{Si}$ to the $\mathrm{Er}$ in the $800-\mathrm{nm}$ wavelength region [13]. However, we point out in this letter that this energy transfer route may be highly problematic, due to an excited state absorption transition that is known from early work on erbium-doped fiber amplifiers (EDFAs).

The basic reason why $800-\mathrm{nm}$ excitation of erbium is unattractive can be seen from Fig. 2: There are transitions at and around this energy (labeled VI in the figure) that can cause the Er metastable $\left({ }^{4} I_{13 / 2}\right)$ state to be excited directly to higher level $\left({ }^{4} S_{3 / 2},{ }^{2} H_{11 / 2}\right)$ states. Excitations to these states serve no useful purpose, but merely pose a debilitating energy drain: EDFAs pumped at $800 \mathrm{~nm}$ require about an order of magnitude more pump power to achieve the same gain as at $980 \mathrm{~nm}$, a level which suffers from no excited state absorption [14]. It is the purpose of this letter to point out that a similar interaction should be occurring in many of the Si nc-Er materials reported, with similarly adverse impact.

We consider the behavior of the Si nc sensitised Er system via a rate equation analysis similar to previous reported models [8], 


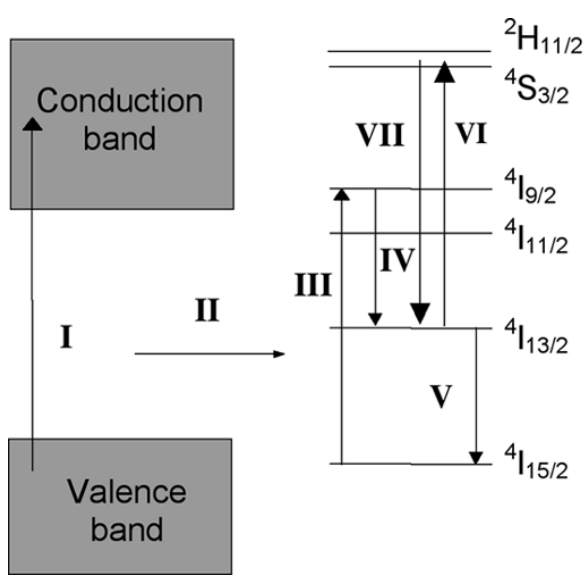

Fig. 2. Model of the Si nc-Er material system, including the excited state energy transitions.

but which takes into account the excited state absorption. The rate equation for the $\mathrm{Si} \mathrm{nc}$ can be written as

$$
\frac{d n_{b}}{d t}=\sigma_{a b} \phi\left(n_{0}-n_{b}\right)-\frac{n_{b}}{\tau_{b}}-\sum_{i=1}^{2} C_{b i} n_{b} N_{i}
$$

where $n_{b}, n_{a}$ represent the upper and lower Si nc population levels with $n_{a}+n_{b}=n_{o}, \sigma_{\mathrm{ab}}$ is the nc absorption cross section and $\phi$ the pump photon flux. $\tau_{b}$ is the Si nc upper state recombination time, $C_{b 1}$ and $C_{b 2}$ are the Si-Er energy transfer coefficients for transitions III and VI in Fig. 2, and $N_{1}$ and $N_{2}$ are the Er population densities in the ground $\left({ }^{4} I_{15 / 2}\right)$ and metastable $\left({ }^{4} I_{13 / 2}\right)$ states, respectively. In the steady state, the Si nc PL behavior can be written as

$$
\text { Si nc PL } \propto n_{b}=\frac{\sigma_{\mathrm{ab}} \phi n_{0}}{\sigma_{\mathrm{ab}} \phi+\frac{1}{\tau_{b}}+C_{b 1} N_{1}+C_{b 2} N_{2}} \text {. }
$$

We can assume to a good approximation that the relaxation processes corresponding to transitions IV and VII are rapid in comparison to both the Er metastable and $\mathrm{Si}$ nc recombination rates (the nonradiative decay times from the ${ }^{4} S_{3 / 2}$ and ${ }^{2} H_{11 / 2}$ levels down to the ${ }^{4} I_{9 / 2}$ and ${ }^{4} I_{11 / 2}$ levels are $\sim 1 \mu$ s [14]. For scientific clarity, we limit consideration to the case of relatively low erbium ion concentrations $\left(<10^{19} \mathrm{~cm}^{-3}\right)$; this allows us to separate effects due to excited state absorption from complications due to Er clustering effects, such as cooperative upconversion and concentration quenching. Finally, we note that the Er metastable lifetime is at least an order of magnitude longer than the Si nc counterpart (several ms versus $100 \mu \mathrm{s}$ ). Therefore, even at relatively low pump powers $\left(0.5 \mathrm{~W} / \mathrm{cm}^{2}\right.$ at $\left.488 \mathrm{~nm}^{2}\right)$, the $\mathrm{Er}$ ions will be in saturation. Under these conditions, we will have $N_{1} \sim 0$ and $N_{2} \sim N_{\mathrm{Er}}$, where $N_{\mathrm{Er}}$ is the total number of $\mathrm{Er}$ ions. Equation (2) then simplifies readily to

$$
\text { Si nc PL } \propto \frac{\sigma_{\mathrm{ab}} \phi n_{0}}{\sigma_{\mathrm{ab}} \phi+\frac{1}{\tau_{b}}+C_{b 2} N_{\mathrm{Er}}} .
$$

It is not difficult to show that the corresponding Er 1550-nm PL can be written as

$$
\operatorname{Er} \mathrm{PL} \propto \frac{N_{2}}{\tau_{\mathrm{Er}}}=C_{b 1} n_{b} N_{1}
$$

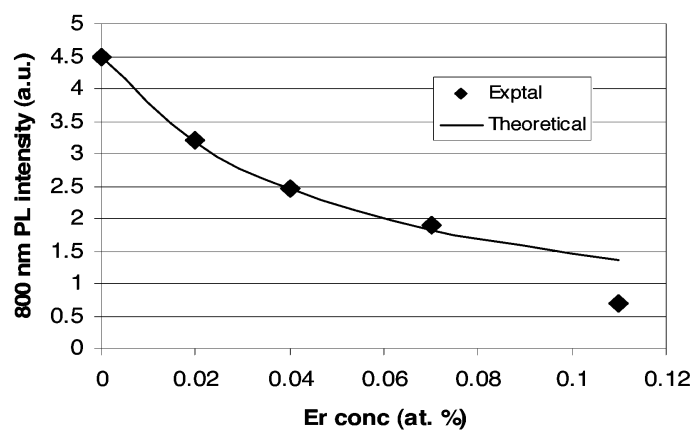

Fig. 3. Predicted Si nc PL behavior with Er concentration (exptal data from [15]).

\section{RESUlTS AND DISCUSSION}

From the above relationships, we note the following behavior: The Si nc PL increases directly with the Si nc concentration, but decreases in an inverse manner with the Er ion concentration. The Er 1550-nm PL, on the other hand, is driven simply by the Si nc-Er energy transfer through the Er ground state, and is effectively oblivious to activity relating directly to the excited state energy transfer, other than through reduced efficiency.

It is useful to compare the behavior described by (3) with data already reported in the existing literature. A substantial decrease in the Si nc PL with increasing Er ion concentration has often been observed [4], [8], [15], but to the authors' knowledge, the correct reason for this behavior has yet to be satisfactorily provided. The confusion may be due in part to the fact that much of the work in this area has involved samples with high Er ion concentrations $\left(\sim 10^{20} \mathrm{~cm}^{-3}\right)$; the overall PL behavior is then masked by Er cooperative upconversion effects. Thus, in the work of Pacifici et al. [8], the model considered cooperative upconversion but ignored excited state absorption. While reasonable agreement with experiment could be claimed for high Er concentrations where ion clustering effects dominate, their predicted Si nc PL behavior for low Er concentration $\left(10^{18}-10^{19} \mathrm{~cm}^{-3}\right)$ is basically flat, largely independent of Er concentration (Fig. 3 of [8]), in contrast to observed erbium-related quenching of nc PL even for concentrations $<10^{19} \mathrm{~cm}^{-3}$. From a physical viewpoint, it is not difficult to see why: In the absence of excited state absorption and ion clustering, the $\mathrm{Er}$ ions are easily driven into saturation, and are overwhelmingly in the metastable $\left({ }^{4} I_{13 / 2}\right)$ state. The steady state transfer of energy from the Si nc to the Er ions under these circumstances would be very small, restricted by the long (ms) Er metastable state decay time. With the Si nc-Er energy transfer route effectively shut off due to Er saturation, it is largely left to the much faster radiative recombination of the $\mathrm{Si}$ nc to dissipate the pump energy. Changes in the Er ion concentration would therefore have only a minor impact on the Si nc PL under the previous model.

With the inclusion of Er excited state absorption, the impact of Er concentration on the Si nc PL is much greater, as the Er ions act as a constant drain of energy from the Si nc by cycling through transitions VI and VII. The Si nc PL behavior at low Er concentrations was carefully characterized by Fujii et al. [15], and we compare our model to their data here. Fig. 3 shows the predicted behavior from (3), with the experimental data from 
[15]. We see that there is quite good agreement, with some deviation occurring for the highest Er concentration data point that could be due to the onset of Er ion clustering. We emphasize that without excited state absorption, it is difficult to account for the observed large decreases in the Si nc PL. The fit also enables us to estimate the value of $C_{b 2}$, the $\mathrm{Si}-\mathrm{Er}$ energy transfer coefficient for the excited state transition. We obtain a value for $C_{b 2}$ of $1 \times 10^{-15} \mathrm{~cm}^{3} / \mathrm{s}$, comparable in magnitude to the Si-to-Er ground state energy transfer coefficient $C_{b 1}$, which has previously been determined [8] at $3 \times 10^{-15} \mathrm{~cm}^{3} / \mathrm{s}$. The energy drain posed by this excited state transition is, therefore, of more than just minor significance, and should not be ignored in the design of this material system. Finally, it is worth noting that our finding is also consistent with the early work from EDFAs: Under 800-nm excitation, the Er excited state absorption cross section was measured to be comparable to the ground state absorption cross section as well.

\section{CONCLUSION}

In summary, to achieve efficient gain operation and viable optical amplifiers and lasers, the Si nc-Er material system should be optimized in order to avoid the Er excited state transition associated with resonant energy transfer at the $1.5-1.6 \mathrm{eV}$ (800-nm wavelength) region. This might not prove to be too difficult, once it is recognized as an issue: Recent work on shifting the luminescence spectra of Si nanocrystals indicates that doping with phosphorus or boron may be a simple but effective means of bandgap engineering the peak wavelength of the Si nc PL spectrum [16]. If the Si nc PL can be shifted to the $980-\mathrm{nm}(1.2 \mathrm{eV})$ region, the prospects for efficient optical amplifier and laser operation based on the $\mathrm{Er}-\mathrm{Si} \mathrm{nc}$ material system could be boosted considerably.

\section{REFERENCES}

[1] J. Lee, J. H. Shin, and N. Park, "Optical gain at $1.5 \mu \mathrm{m}$ in nanocrystal Si-sensitized Er-doped silica waveguide using top-pumping $470 \mathrm{~nm}$ LEDs," J. Lightw. Technol., vol. 23, no. 1, pp. 19-25, Jan. 2005.
[2] A. Polman and F. C. J. M. van Veggel, "Broadband sensitizers for erbium-doped planar optical amplifiers," J. Opt. Soc. Amer. B, vol. 21, pp. 871-892, 2004.

[3] A. J. Kenyon, P. F. Trwoga, M. Federighi, and C. W. Pitt, "Optical properties of PECVD erbium-doped silicon-rich silica: Evidence for energy transfer between silicon microclusters and erbium ions," J. Phys: Condens. Matter, vol. 6, pp. L319-L324, 1994.

[4] M. Fujii, M. Yoshida, Y. Kanzawa, S. Hayashi, and K. Yamamoto, "1.54 $\mu$ m photoluminescence of $\mathrm{Er}^{3+}$ doped into $\mathrm{SiO}_{2}$ films containing Si nanocrystals: Evidence for energy transfer from Su nanocrystals to $\mathrm{Er}^{3+}, "$ Appl. Phys. Lett., vol. 71, pp. 1198-1200, 1997.

[5] F. Iacona, Di. Pacifici, A. Irrera, M. Miritello, G. Franzo, F. Priolo, D. Sanfilippo, G. D. Stefano, and P. G. Fallica, "Electroluminescence at $1.54 \mu \mathrm{m}$ in Er-doped Si nanocluster-based devices," Appl. Phys. Lett., vol. 81, pp. 3242-3244, 2002.

[6] H.-S. Han, S.-Y. Seo, and J. H. Shin, "Optical gain at $1.54 \mu \mathrm{m}$ in erbiumdoped silicon nanoclusters sensitized waveguide," Appl. Phys. Lett., vol. 79, pp. 4568-4570, 2001.

[7] A. J. Kenyon, C. E. Chryssou, C. W. Pitt, T. Shimizu-Iwayama, D. E. Hole, N. Sharma, and C. J. Humphreys, "Luminescence from erbiumdoped silicon nanocrystals in silica: Excitation mechanisms," J. Appl. Phys., vol. 91, pp. 367-374, 2002.

[8] D. Pacifici, G. Franzo, F. Priolo, F. Iacona, and L. Del Negro, "Modeling and perspectives of the Si nanocrystals-Er interaction for optical amplification," Phys. Rev. B, vol. 67, pp. 245 301-245 313, 2003.

[9] F. Gourbilleau, M. Levalois, C. Dufour, J. Vicens, and R. Rizk, "Optimized conditions for an enhanced coupling rate between Er ions and $\mathrm{Si}$ nanoclusters for an improved $1.54 \mu \mathrm{m}$ emission," J. Appl. Phys., vol. 95 , pp. 3717-3722, 2004.

[10] M. V. Wolkin, J. Jorne, P. M. Fauchet, G. Allan, and C. Delerue, "Electronic states and luminescence in porous silicon quantum dots: The role of oxygen," Phys. Rev. Lett., vol. 82, pp. 197-200, 1999.

[11] A. Puzder, A. J. Williamson, J. C. Grossman, and G. Galli, "Surface chemistry of silicon nanoclusters," Phys. Rev. Lett., vol. 88, pp. 097 401-097 404, 2002.

[12] A. J. Kenyon, P. F. Trwoga, C. W. Pitt, and G. Rehm, "The origin of photoluminescence from thin films of silicon-rich silica," J. Appl. Phys., vol. 79, pp. 9291-9300, 1996.

[13] K. Watanabe, M. Fujii, and S. Hayashi, "Resonant excitation of $\mathrm{Er}^{3+}$ by energy transfer from Si nanocrystals," J. Appl. Phys., vol. 90, pp. 4761-4767, 2001.

[14] E. Desurvire, Erbium Doped Fiber Amplifiers. New York: Wiley, 1994.

[15] M. Fujii, M. Yoshida, S. Hayashi, and K. Yamamoto, "Photoluminescence from $\mathrm{SiO}_{2}$ films containing $\mathrm{Si}$ nanocrystals and Er: Effects of nanocrystalline size on the photoluminescence efficiency of $\mathrm{Er}^{3+}, " J$. Appl. Phys., vol. 84, pp. 4525-4531, 1998.

[16] M. Fujii, K. Toshikiyo, Y. Takase, Y. Yamaguchi, and S. Hayashi, "Below bulk-band-gap photoluminescence at room temperature from heavily P-and B-doped Si nanocrystals," J. Appl. Phys., vol. 94, pp. 1990-1995, 2003. 